\title{
Self-healing research of ZigBee network based on coordinator node isolated
}

\author{
Ming-Jiang Huang \\ School of Automation \\ Beijing Institute of Technology, \\ Beijing, China \\ Email: hmj110131@sina.com
}

\author{
Tao Wang \\ School of Automation \\ Beijing Institute of Technology \\ Beijing, China \\ Email: WangTaobit@bit.edu.cn
}

\begin{abstract}
A single-chip solution is proposed to realize the node design based on $2.4 \mathrm{GHz}$ ZigBee wireless sensor network. Mesh network topology is achieved by the use of ZigBee Pro protocol stack on the hardware platform. When the packets are successfully sent to the coordinator node, a relevant event will be generated by the local node. Hence, the parent node lost event can be obtained by comparing the number of the packets sent by the child node and the number of the packets sent successfully shown by the respond event. A wireless sensor network is designed to work independently from the coordinator node and can be re-connected according to the needed set to resume the network. When the coordinator node is taken away from the site or power down by happenstance and power up again after a long time, the parent lost event in the child nodes can make it rejoin the new coordinator node again when needed. The network self-healing function can be achieved by this mechanism that makes the network more stable.
\end{abstract}

\section{Keywords-JN5148; ZigBee Pro; mesh; self-healing network}

\section{INTRODUCTION}

With the depth theoretical study of wireless sensor networks and new emerging IC technology, wireless sensor networks mining in various fields of application value. WSN has been increasingly used in military and civilian fields (such as battlefield surveillance, security, and disaster management, etc.), the key technologies of wireless sensor networks is becoming today hotspot [1]. ZigBee is a lowcost, low-power, low-data rate, short-range wireless network technology [2].

The current monitoring system based on wireless sensor networks that mostly used for a long-term monitoring. Sensor node devices fixedly mounted on the site environment, the acquired data will be transmitted through wireless sensor networks to the coordinator node, and then via a wired or wireless transmission to the server for processing and analysis. The similar systems are often high cost, maintenance is difficult, and the node installation is not flexible enough. Sending the data of the coordinator node to the monitoring server by GPRS, Internet or other transmission means, the overall system will be more complex, and also will be affected by the impact of the unstable factors such as the internet's link quality [3].

In some acquisition system, the sensor nodes fixed installation in the field. Coordinator node only when needed to start the network services, that is often need to restart all the child nodes one by one to re-establish a network connection. In addition, the coordinator node's installation is inconvenient sometimes, even need to leave the scene for a long time. In such systems, the installation position of the coordinator, power security and the portability of the coordinator node that have become a problem which cannot be ignored. For this kind of problem, a restart mechanism which monitors coordinator node network lose event, can let the child nodes in each hop work independently, and achieve fast self-recovery of the network connection when the coordinator node demand to restart the networks, which helps to enhance the portability of the coordinator node and system reliability.

\section{HARDWARE DESIGN OF THE NETWORK NODES}

The design of the network nodes, include power management, data acquisition, data storage and wireless transmission of four parts. Power supply with battery supply and field supply two alternative methods, while monitoring the status of the battery power consumption on the node.

A microcontroller unit is always used as the core part of the data acquisition system. And the inner ADC in MCU or external expansed one will be used to acquire the sensor data for sampling, quantization and coding. The digital signal then will be sent to the wireless transmission unit by a uart port.

In wireless data transmission part, a 32-bit wireless microcontroller JN5148 is used as the core unit for the data packaging and sending it to the coordinator node. Jennic has introduced the third generation of ZigBee single-chip JN5148, which can support the highest version ZigBee communication protocol. In ZigBee single-chip wireless microcontroller market, JN5148 can provide maximum memory capacity of 4 Mbit and the lowest power consumption without PA. Its receive current consumption is $15 \mathrm{~mA}$, the receiver sensitivity is-98dBm, the emission current is $17 \mathrm{~mA}$, emission power consumption is $20 \mathrm{dBm}$, the data bandwidth is $250 \mathrm{Kbps}$, power consumption is at least $25 \%$ lower than existing solutions on the market [4]. JN5148 high-gain module, the transmission distance of point to point can reach of farthest visual distance between $1000 \mathrm{~m}$ and effectively to meet the data transmission distance. Figure 1 is a block diagram of sensor node hardware.

\section{NETWORKING AND SELF-HEALING RESEARCH}

Establishing a mesh structure network based on the ZigBee Pro protocol stack, then the child node will site environment for suitable choice of routing nodes relay, and eventually upload all child nodes of the sensor data to the coordinator and the processed data will be sent to the site 
server monitoring software to achieve the real-time processing and analysis [5].

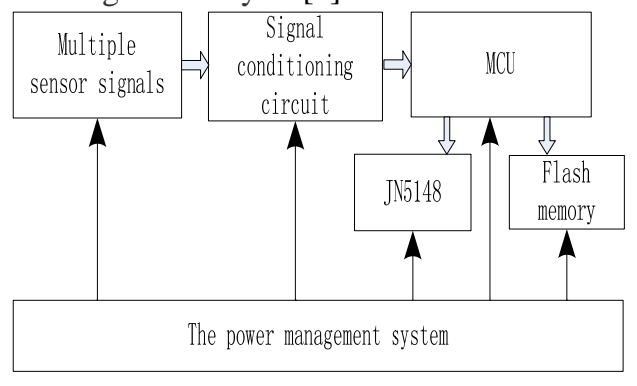

Figure1. ZigBee node hardware structure diagram

\section{A. Establishment of mesh network}

The networking process of different nodes is different in ZigBee Pro protocol stack [6]. In the coordinator node, after power-up, first to initializate the system, and then process to examine the current environment of idle RF channel if it detects the channel is not occupied or not in busy, it will receive a response signal, otherwise jump to the next channel to continue testing. The coordinator node in the current channel to start protocol stack, call functions ZPS_eAplZdoStartStack, to initiate the network, begins to enter the network listening state. When there have the request that nodes apply to join, the function PRIVATE void vHandleStackEvent (ZPS tsAfEvent sStack Event) will be called to produce the corresponding event. After determine the type of the node by the event, if that is not the coordinator node, nodes with the same PanID that will be allowed to join. At the same time, a local ACK response will be generated, determining the network time-out response, and update local address link table. Coordinator node set up a network flow chart shown in Figure 2.

The process of router nodes and terminal nodes join the network and the process of coordinator node start networks is not the same, which is mainly applied to join the network of existed that coordinator node initiated, without any impact on the network parameters. After child node is powered up and initialization is completed, according to the network configuration parameters, searching channels that be configurated, if the channel has been launched by the coordinator node, the child node will request to join it and wait for the response that coordinator node has accepted the apply. When this node receives the event, indicate that the communication has been established, the next step work in the application layer is the process to send and receive application data. If the network request to join the network, but the response is not generated, or response timeout, the system will repeat to send network join request, the cycle to restart the protocol stack until the establishment of communication. All network child nodes join the network process is shown in Figure 3.

\section{B. Network self-healing function}

In the wireless sensor networks due to anthropogenic causes may cause the power-down of the coordinator or artificially restart, or the causes of accidents caused by poor link quality caused by the loss of a parent node link, the child nodes cannot continue to communicate with their parent node. The network architecture of mesh topology can guarantee that when the child node and its routing parent node communication link is broken, searching other router nodes for data relay. However, on-site installation and other reasons, the child of the networks cannot still connect to the old coordinator node. When the coordinator node after a long time and re-power on or returned to the scene again, all the sub-node has been disconnected with the coordinator node, so there is a need to re-discover and rejoin the new coordinator node. As nodes shown in Figure 4 and Figure 5.

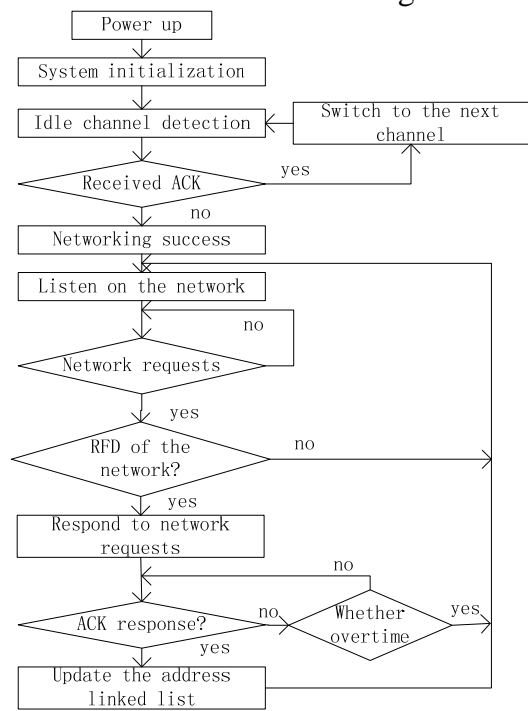

Figure 2.The flow of Coordinator to initiate a network Power up

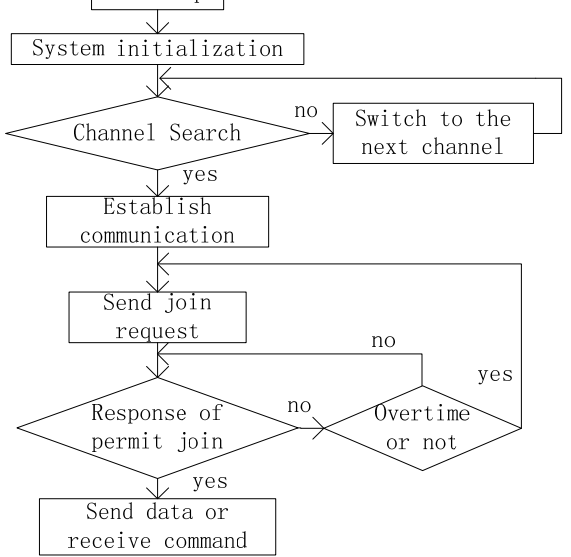

Figure3. The flow of routers and terminals join the network

1) ZigBee Pro protocol stack provide a random address allocation mechanism, when the parent node after power up or reset, the saved original network parameters of sub-node can no longer be consistent with the new network. For this problem existed in the ZigBee Pro protocol stack, depending on the operating mode of the node in the application layer program, a combination of two methods to detect the lost event of the parent node to restart the child node's stack. The child node will get a new network identity to re-apply to join 
the network after this event, and obtain a new 16 network address. Timing call coordinator

JenOS operating system is a real-time multitasking operating system that Jennic introduced for JN5148 and ZigBee Pro protocol stack to the organic combination. Task scheduling unit and event management unit to complete the unified management of hardware resources and the ZigBee Pro protocol stack network event. JenOS Includes: real-time task scheduling systems, data storage management unit, power management control unit, protocol stack data communication control unit and software debug system unit.

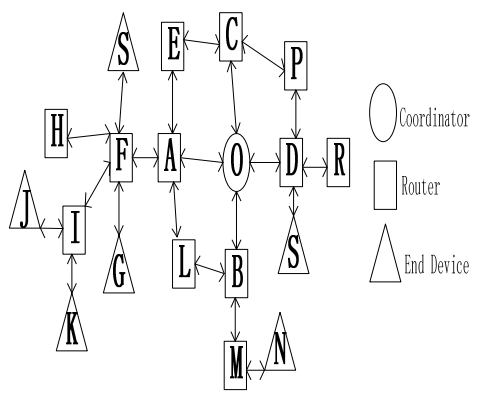

Figure 4.The network structure of the system that works properly.

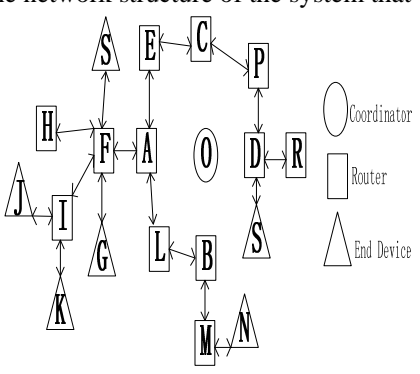

Figure 5.The network without the coordinator node.

According to the execution characteristics of JenOS operating system , during the task execution in the child nodes timed call the parent node, to achieve the detection of the parent node is online or not. Power-down when the parent node or link is lost, reboot the stack of the local node until its parent node or link is restored, and then create a new connection. The execution steps of the method are as follows.

a) Build a software timer in the child node, the time value is defined as timer_time. When the implementation of the task, executing the timer, and last send a certain length of custom data to the parent node.

b) In application layer program of the child nodes, set a sign OS_Run_Num to count the task execution times, the number that a coordination node successfully received data packet define as ACK_Num.

c) Get the parent node lose events. When the task executive number (the number of timing calls), much larger than the number of the ACK response in the local event (ZPS_EVENT_APS_DATA_ACK) that indicate the sensor data has received by the destination node. Which can be concluded that the parent node is lost that maybe caused by power down or the link quality is not sufficient to establish a stable connection, indicating that the network need to reconnect.

d) In step 3, "much larger", assuming as ACK_Lose_Num. Task execution speed is determined by the internal code length, there also happens to be the case that the network indication event missed in the task execution process, but the next execute time the task will capture this event. Therefore, there is a following relationship.

$$
\text { OS_Run_Num }>\text { ACK_In_Num + ACK_Lose_Num }
$$

The value of ACK Lose Num in the formula should be set reasonable based on the actual situation. When the value is too small, which may be due to the packet lose that caused by network link poor quality, resulting in fallacious judgement of network events and restart the network and modify the network parameters. The value should not be set too big, otherwise, the coordinator power up after power down, will wait a long time to reconnect to their child nodes.

Child nodes that need to reconnect to their parent node by restart protocol stack. Based on the need to set the size of the value of ACK_Lose_Num to thereby obtain the child nodes restart time. Network re-connection time is defined as restart_time, then:

restart_time $>$ ACK_Lose_Num $\times$ timer_time

e) When meet the above conditions, the need to restart the protocol stack, at the same time, the non-volatile memory within the network parameter information should be cleared. Child nodes will get the identity of the new node to re-join the network until the coordinator reconnected or link recovery, allocated by the coordinator node to obtain a new network parameters.

2) Serial packet ACK event

When the continuous transmission of sensor data, the send time interval ( $t x \_p k t$ time) can be controlled by the MCU serial port, tx_pkt_time in this system is set to 1 second, then JN5148 will send out the received serial data immediately.The function of ZPS_eAplAfUnicastAckDa-

taReq is used to send the data,then the local node will generate a event (ZPS_EVENT_APS_DATA_ACK) that respond automaticly by the destination node to show that the destination node has successfully received the sensor data. Packets successfully received by the parent node, then it will return a corresponding ACK message to the source node and produce a ZPS_EVENT_APS_DATA_ACK event in the source node, to inform the data has successfully reached the parent node. But when the link quality is not good, there packet cannot reach to the parent node or the parent node is unable to respond a ACK message to the source node, resulting in packet loss. In this case, the above function will be called once again to send the packet. Three times in a row is still not receive a confirmation signal ACK events, and then the data will be discarded, and change to send a new packet. Based on this mechanism, combined with the MCU's serial port to send the sensor data packet rate and check the 
number of the ZPS_EVENT_APS_DATA_ACK events to obtain the lost event of the parent node. The executive steps are as follows.

a) The asynchronous serial port of JN5148 listen sensor data from the MCU in real time, calibration data and obtaining the length of the packet, according to the MCU serial port transmit frequency to obtain the approximate packet transmission interval, add the cumulative number of packets sent as tx_num.

b) When the sensor data packet has been successfully transmitted and received by the Coordinator node, a response message will reply to the source node and the source node will produce a ZPS_EVENT_APS_DATA_ACK event. When the event is generated, add a cumulative number of responses that set as tx_ack_num, the last response number is set as tx_ack_num0.

c) Since the child nodes under the normal working conditions is in a continuous transmission status, exclude the packet loss situation, the response number of the packet that successfully transmitted should be substantially equal to the number of packets sent by the source node. Therefore, comparing the successfully transmitted packet number and the number of the response, while judge the ACK response is whether or not changed, if no changes and no ACK response occurs, in this case, which can be concluded that the parent node has lost or the network link is not stable.

d) When the parent node has lost or link disconnected, according to the parent node restart or link recovery time interval to determine the restart mechanism. According to the time interval of the sent packets, and the number of the lost ACK (ACK_Lose_Num), the restart time can be get, which defined as restart_time. Therefore, there is a following relationship.

$$
\text { restart_time }=t x_{-} \text {pkt_time } \times \text { ACK_Lose_Num }
$$

e) When the coordinator is powered down or the link is disconnected, the child node to send the data packets will be lost, and the difference between the number of the packets sent and the number of the response generated will gradually become larger. When the value is greater than ACK_Lose_Num, child will restart the protocol stack, waiting for the coordinator node re-power on to recover or link to reconnect until the network reconnect successfully. The node in this process will get a new network identity, and the network parameters will be assigned again by the coordinator.

\section{Experimental verification}

These two Combining methods can obtain the loss event of coordinator node. When there have data come from the serial port, the second method can be used to detect the parent node lost event. When the host computer control the child node to stop data acquisition, or enter into the offline acquisition mode, then use the first method timing call the coordinator to get the online status of the parent node.
Sensor data packet is controlled by MCU, when set to 1 second to send once. The above parameters ACK_Lose_Num then be set to 60 , according to the formula, the restart time is set to 1 minute. Also, when the timer interrupt time is set to 1 second, the restart time is set to 1 minute. When data is sent continuously, packets have lost, until the lost packet number is larger than ACK_Lose_Num, then believes that the contact of the child node and parent node has been disconnected, there have need to restart the network. After about 60 second, the child node will restart the protocol stack and then rejoin the network as a new identity and get a new 16-bit short address, whenever the coordinator node power up again or link recovery again. Like the above method, the child node can restart the protocol stack after above 60 seconds, and rejoin the coordinator whenever power up again. After waiting a long time, the coordinator node is power-up, the child node will re-scan the channel, and continue to restart the protocol stack until it joined the new network. Large numbers of experimental verification shows that the protocol stack restart time error is maintained at less than $5 \%$, verified by setting different restart time. The rate of success recovery of the network is of more than $90 \%$. Some of the experimental data in Table 1 below lists.

TABLE I THE EXPERIMENTAL DATA OF NETWORK RESUME

\begin{tabular}{|c|c|c|c|c|}
\hline $\begin{array}{c}\text { Number } \\
\text { of } \\
\text { restarts }\end{array}$ & $\begin{array}{c}\text { ACK_Lose_Num } \\
\text { value }\end{array}$ & $\begin{array}{c}\text { Average } \\
\text { restart } \\
\text { time } \\
\text { (seconds) }\end{array}$ & $\begin{array}{c}\text { Timing call } \\
\text { coordinator } \\
\text { successful } \\
\text { times }\end{array}$ & $\begin{array}{c}\text { Serial data } \\
\text { call } \\
\text { coordinator } \\
\text { successful } \\
\text { times }\end{array}$ \\
\hline 10 & 60 & 60 & 10 & 10 \\
\hline 10 & 600 & 602 & 10 & 10 \\
\hline 10 & 3600 & 3622 & 10 & 10 \\
\hline 10 & 36000 & 36350 & 10 & 10 \\
\hline 10 & 72000 & 72600 & 10 & 10 \\
\hline
\end{tabular}

\section{CONCLUSION}

A JN5148 chip is used to design a low power hardware platform, and the ZigBee Pro protocol stack is used to establish a mesh structure network topology. When the coordinator node is power-down, and after a long time, restart the coordinator node again. The program can improve the network's ability to repair itself. That ensures the stability of the system in which the field application, avoiding coordinator node's power-down caused due to man-made and other reasons that need to restart all child nodes. The method has a very important practical significance. Does not only the wireless sensor monitoring networks can be applied, but also when there is a need to consider the multi-sensor nodes system's reliability, the program can provide some theoretical support.

\section{REFERENCES}

[1] Zhang Hong-Feng, Li Wen-Feng. Wireless sensor networks based on ZigBee technology research [J].Wuhan University of Technology (Information \& Management Engineering), 2006, (08).

[2] Li Jianzhong, Gao Hong. The high macro wireless sensor network research progress [J]. Computer Research and Development, 2008,45 
(1) :1-15.

[3] Wang Shu, wireless sensor networks theory [M] Beijing: Beijing University of Aeronautics and Astronautics Press, 2007.

[4] JNS148Datasheet(EB/OL).http://www.jennie.com/support/datasheet/ jn5148_wireless_microcontroller_datasheet. 2010.

[5] Zhang Qiwei, Bridge Health Monitoring concepts and monitoring system design [J]. Shanghai: Tongji University, 2001 (1) 66-69.

[6] Zhou Lin, Chen Yu, Feng Ting-ting, Self-healing Network Organization and Protocol Implementation based on ZigBee Technology [J]. Beijing,

Communications Technology,Vol. 45, No. 04, No. 244, Totally, 2012. 